\title{
Neues Medikament schützt MS-Patienten schon nach dem ersten Schub
}

Die beiden Zulassungsstudien TEMSO und TOWER haben bereits demonstriert, dass sich bei MS-Patienten mit dem neuen oralen Medikament Teriflunomid sowohl die Schubrate als auch die Behinderungsprogression deutlich reduzieren lässt. Offenbar kann das Arzneimittel auch bei Patienten mit einem ersten klinisch isolierten Schub die Konversion zu einer klinisch manifesten MS aufhalten. Dazu stellte Professor Patrick Vermersch, Uniklinik Lille/Frankreich, erste Daten der TOPIC-Studie vor. An der Studie nahmen 618 Patienten mit einem ersten demyelinisierenden Ereignis teil. Je etwa ein Drittel von ihnen bekam Placebo, $7 \mathrm{mg} / \mathrm{d}$ oder $14 \mathrm{mg} / \mathrm{d}$ Teriflunomid. Primärer Endpunkt war die Konversion in eine klinisch manifeste MS während der zweijährigen Studiendauer.
Unter Teriflunomid $7 \mathrm{mg}$ war die Konversionsrate um $37 \%$ geringer als unter Placebo, in der Gruppe mit $14 \mathrm{mg}$ sogar um $43 \%$. Therapieabbrüche aufgrund unerwünschter Wirkungen erfolgten in allen Gruppen ähnlich häufig $(9,1 \%$ unter Placebo, $12,1 \%$ bzw. $8,3 \%$ mit 7 bzw. $14 \mathrm{mg}$ Teriflunomid). Insgesamt, so Vermersch, deute das umfangreiche Studienprogramm zu Teriflunomid mit über 5.000 Patienten und zum Teil über zehn Jahren Beobachtungszeit auf ein sehr gutes Sicherheitsprofil hin.

Auch bei einer Langzeittherapie mit dem Medikament sei es nicht zu einer erhöhten Rate von Tumoren oder schweren Infektionen gekommen. Blutbildanalysen hätten auch keine Hinweise auf eine Immunsuppression ergeben: Zwar sinke die Zahl der Lymphozten und neutrophilen
Granulozyten mit der Therapie um etwa $15 \%$, die Werte blieben in der Regel aber in normalen Bereichen. Etwas häufiger als mit Placebo kam es in Studien unter Teriflunomid zu Durchfall, Übelkeit, verminderter Haardichte und Leberwerterhöhungen. Die Effekte seien aber in der Regel leicht bis moderat gewesen und waren meist auf die ersten Therapiewochen und -monate begrenzt.

In den USA und Australien ist Teriflunomid (Aubagio ${ }^{\circ}$ ) bereits zugelassen. Die Europäische Arzneimittelagentur (EMA) hat eine positive Stellungnahme abgegeben. Thomas Müller, Springer Medizin

Satellitensymposium „Current challenges of MS therapy: accepted". Kongress der European Neurological Society (ENS) in Barcelona, 10.6.2013 Veranstalter: Genzyme - A Sanofi Company

\section{Neuropsychiatrische Symptome und kognitive Fähigkeiten verbessert}

In einer randomisierten placebokontrollierten Doppelblindstudie mit Patienten, die eine amnestische leichte kognitive Störung (amnestic Mild Cognitive Impairment, aMCI) aufwiesen, verbesserte sich während der Behandlung mit dem Ginkgo-Spezialextrakt EGb $761^{\circledR}$ (Tebonin $^{\circledast}$ ) nicht nur die kognitive Funktion. Auch beim Gesamtscore des Neuropsychiatrischen Inventars (NPI) sowie der NPI-Domäne Angst zeigte EGb $761^{\circ}$ einen signifikanten Vorteil gegenüber Placebo.

Professor Ulrich W. Preuss, Psychiatrische Klinik am Kreiskrankenhaus Prignitz, Perleberg, stellte in Nizza Teilergebnisse der Multicenterstudie mit 160 Patienten vor. Die Patienten litten an aMCI nach den internationalen Konsensuskriterien von Winblad et al. (2004) und wiesen einen Wert von mindestens sechs
Punkten auf dem Gesamtscore des NPI auf. Patienten mit Demenz, manifesten psychischen oder schweren körperlichen Erkrankungen wurden ausgeschlossen, ebenso Psychopharmaka als Begleitmedikation - mit Ausnahme von gelegentlich eingenommenen kurz wirksamen Benzodiazepinen und Baldrianpräparaten gegen Schlafstörungen.

Die Patienten wurden randomisiert doppelblind entweder mit $240 \mathrm{mg} / \mathrm{d} \mathrm{EGb}$ $761^{\circledast}$ oder Placebo über einen Zeitraum von 24 Wochen behandelt. Der durchschnittliche Gesamtscore auf der NPIZwölfpunkteskala lag in der Placebogruppe initial bei 11,6 \pm 3,7 und ging während der Behandlung um 5,5 \pm 5,2 Punkte zurück. In der Verumgruppe betrug er initial $11,5 \pm 3,5$ und ging um 7,0 \pm 4,5 Punkte zurück; dieser Unterschied zwischen den beiden Behandlungsarmen ist statistisch signifikant $(p<0,001)$. Eine Gesamt-NPI-Verbesserung um mindestens vier Punkte - ein im Studiendesign vordefinierter Zielparameter - wurde bei $79 \%$ der Patienten unter Verum und bei $56 \%$ der Patienten unter Placebo gefunden. Die Analyse der Einzeldomänen des NPI ergab eine statistisch signifikant stärkere Reduktion von Angstsymptomen in der Verum- gegenüber der Placebogruppe. Auch die kognitiven Fähigkeiten (erfasst im Trail Making-Test) verbesserten sich in der EGb $761^{\circ}$-Gruppe signifikant.

Thomas M. Heim, freier Medizinjournalist

Vortrag „Ginkgo biloba extract EGb 761 in mild cognitive impairment with neuropsychiatric symptoms: a randomized placebocontrolled trial" im Rahmen des 21 th European Congress of Psychiatry in Nizza, 7.4.2013 